\title{
Modularization of Feature Information for Marine Diesel Engine Parts
}

\author{
ZHANG Hui, a , ZHANG Sheng-wen ${ }^{1, b}$, ZHAO Pan ${ }^{1, c}$ and ZHANG Jian ${ }^{1, d}$ \\ ${ }^{1}$ School of Mechanical Engineering, Jiangsu University of Science and Technology, Zhenjiang, China

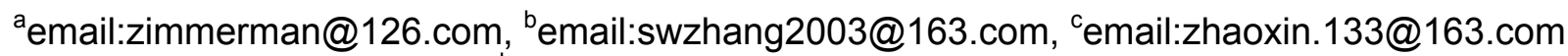 \\ demail:zhangjian_tree@foxmail.com
}

Keywords: modularization; feature information; marine diesel engine; NC tool path generation

\begin{abstract}
According to the modularization principle, focusing on the practical problem of NC tool path generation, feature information is processed with group technology for tooling in CAM (Computer Aided Manufacturing) of the key parts of marine diesel engine. The processing is mainly made on the basis of similarity of the cutting area of the feature, then the information is categorized and the correspondent data are stored in a database. Based on the above research work, a tool path generation software system on UG secondary development platform is developed which can extract necessary tooling information for fast complicated $\mathrm{NC}$ tool path generation. The method is proved to be of great feasibility with validation by Vericut simulation and real machine experiment, which can be beneficial to the tool path generation of other complex parts.
\end{abstract}

\section{Introduction}

Modularization is a high level standardization which is still evolving. It can be defined as each part of a product can be designed individually, and can be combined as one system with small sub-system to perform rather complicated function. Entering the new century, the manufacturing company has featured more varieties and specialized small scale. Modularization has become 21 century's frontier technique, which opened up a new way for standardization work in an era of extraordinary sophistication and versatility.

Diesel engine is important ship propulsion equipment. The marine diesel engine is a typical discrete manufacturing mechanical product which is volume large, structural complex, and manufacturing process miscellaneous. The heavy workload for NC programming requires much manpower and makes it error-prone. So study of one NC tool path generation method for key parts of marine diesel engine has always been a great concern for diesel engine manufacturing company.

\section{Modularization}

In this paper, the feature of key parts for marine diesel engine is focused, analyzed and studied, from the corresponding CAD models. Feature information and processing information are modularized according to group technology, extracted and stored in a database. Based on the modularized and categorized stored information, tool path are generated and then posted to produce NC program. Marine diesel engine key parts CAM system is developed by adopting UG secondary development, and the software is organized modularly and module is also partitioned with reasonability.

Usually modularization is mainly mentioned for big products like shipbuilding, vehicle or aircraft. It is rarely used for manufacturing of component parts such as marine diesel engine. In this paper, the milling regions are

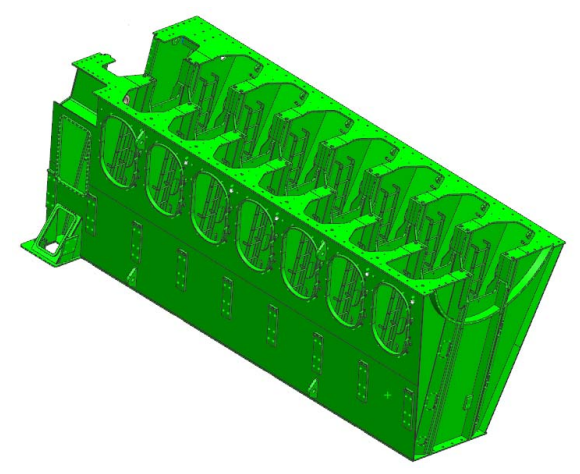

Fig.1 the Frame Parts of Marine Diesel Engine categorized by feature similarity. Through analysis on marine diesel engine frame in Fig.1, we 
can find that frame parts tooling regions are mainly faces, holes, grooves or their combination. They can be categorized as face-like regions like face, side edge, frame face, guide plate face and hole-like regions like cylindrical bore, conical bore, threaded hole, counter bore, hole chamfering. The categorization would have effect on milling manner. There are several kinds of available general purpose CAM software such as UG, MasterCAM. But to use general purpose CAM software directly has such disadvantages: first, process information transmission inconvenient, current general purpose CAM software need huge information by human-computer interaction like tool parameters, main shaft speed, and the information is duplicated among similar milling regions; second, the tool path control manner is limited, because

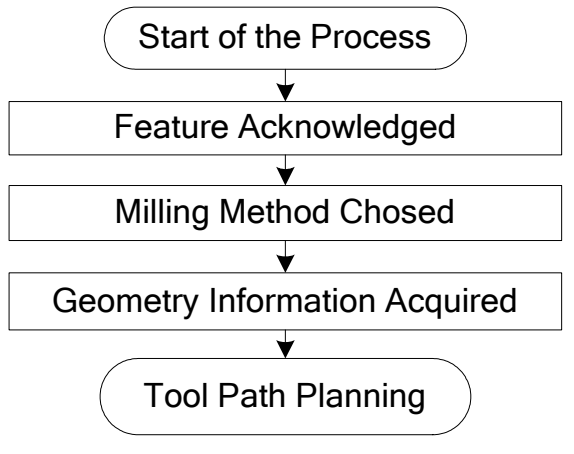

Fig.2 Classic Tool Path Planning Process the marine diesel engine part structural relation is sophisticated, tool path must consider many impact factors, but general purpose CAM software do not provide these input factor interface.

Tool path analysis is seen in Fig.2, the process is to output the tool path based on specific cutting region, part material, cutting position, cutting method, cutting condition aiming to increase the NC programmer's NC programming efficiency. For different milling feature, tool path generation algorithm is different. Marine diesel engine is volume huge, so parts are usually manufactured on large NC boring and milling machine. So the tool path process planning also needs particular care, including tool path stepping.

\section{Similarity Analysis}

During milling, system will generate tool path according to the literally connected path and movement arguments. See in table 2 take doorstep face for example, the system will find the name Uxingmenkuang, and call the tool path function, figure out the cutting argument, like speed of the spindle $600 \mathrm{r} / \mathrm{min}$ feed $200 \mathrm{~mm} / \mathrm{min}$, tolerate $5 \mathrm{~mm}$, the tool number is $\mathrm{T} 01$, and the machine is $14 \mathrm{M}$, auxiliary No. is 4, and at last generate the corresponding tool path. According to the previous structural analysis and process, the main feature to be milling is face-like or hole-like, so after deliberate design and practice of current tool path generation, investigation and summing up, the cutting face manner is listed as follows see in Table1.

Face-like Features. The milling region's boundary information and milling information are required for face-like feature milling like tool parameters and cutting depth. In the milling computation, cutting times depends on the size of the cutting area and the diameter of the tool; cutting depth depends on the face manufacturing degree of precision. The stepping of the tool is normally set as $75 \%$ of the tool diameter

Hole-like Features. If the hole is tiny, the same diameter cutter as the hold can be used to drill. If the hole is big, a small cutter is needed to drill first, then with a big cutter. So the small diameter hole used spot drilling, drilling, big hole with spot drilling and milling. Hole making's parameter is mainly depends on the hole's diameter.

Similarity on Features and Process Information. Through analysis we find that there exist similar or even same features for series of marine diesel parts, their tool path generation rules are almost the same with only different tool changing or tool moving direction. This phenomenon is mainly because of diverse manufacture. By statistics, these similar or same milling features consist about $60 \%$ of all the features. When using general purpose CAM software for NC program, obviously process information for these parts needs to be entered manually, workloads are increased and the efficiency are drained. In this paper, process planning template-prone method is employed to put the similar feature's process grouped, to use same template model for same or similar features. We can find out that not only similarity exists in the different features in one same marine diesel engine, but also between different series of parts. 
Table1 Similarity Information for Milling Regions

\begin{tabular}{|c|c|c|c|c|c|}
\hline Milling Region & $\begin{array}{c}\text { Cutting } \\
\text { Tool }\end{array}$ & $\begin{array}{c}\text { Main Shaft } \\
\text { Speed (r/min) }\end{array}$ & $\begin{array}{c}\text { Feed Rate } \\
(\mathrm{mm} / \mathrm{min})\end{array}$ & $\begin{array}{c}\text { Tolerate } \\
(\mathrm{mm})\end{array}$ & $\begin{array}{c}\text { Auxiliary } \\
\text { No. }\end{array}$ \\
\hline Bottom Face Half-finish & $\varphi 315$ & 200 & 500 & 0.5 & 25 \\
\hline Bottom Face Finish & $\varphi 315$ & 200 & 500 & 0 & 25 \\
\hline Top Face Half-finish & $\varphi 315$ & 200 & 500 & 0.5 & 25 \\
\hline Top Face Finish & $\varphi 315$ & 200 & 500 & 0 & 25 \\
\hline
\end{tabular}

NC Tool Path Post Treatment. After the tool path is generated, post treatment is needed to produce NC program. As for complicated parts as marine diesel engine, aiming to improve the efficiency, process convergence is considered to make "one clamp one station multiple steps", to reduce clamp and tool change times. But the general purpose software cannot consider these factors. So these NC codes need to be joined and edited. The guidelines should be followed: firstly, "face before hole", in one process, milling all faces firstly then drilling all holes; secondly, "machine convergence upon face before hole", use one machine to mill much area at one time, thirdly, "tool convergence upon machine convergence", use one cutter for much cutting work at one time to reduce the tool change times, fourthly, "direction convergence upon tool convergence", in one direction, milling as much as areas as possible to reduce clamp times, fifthly, upon above principles, when the NC programs are joined, amend the joined codes, such as removing the duplicate attachment change, tool change according to the machine requirements.

\section{System Implementation}

Upon above research work, CAM software on UG secondary platform is developed with group technology. In the program, different tool path generation procedures are modularized and categorized and coded.

System Function Division. System function is divided into milling guide and configuration. As seen in Fig.3, tool path generation which is the core function of the software is handled in milling guide; other auxiliary function is provided in configuration which is consisted of process, cutter, machine and post management. All of these are related with the milling guide.

System's software programming is also organization modularization. The overall infrastructure is divided as base data module, database module, tool path generation module, main module, management module and interface related module. All the parameters (cutting argument, main shaft speed, etc.) are defined in the base data module, in the customized form to meet the $\mathrm{C}++$ program standard. Database module is consisted of functions and routines related with database operations, and classes are defined. Tool path generation rules written in $\mathrm{C}$ style language for different feature are included in tool path generation module. The process interface with user is implemented in the main module. All kinds of auxiliary function rely in management module. UG UF/open calls are handled in interface related module, which is in fact the adapter for $U G$ secondary development.

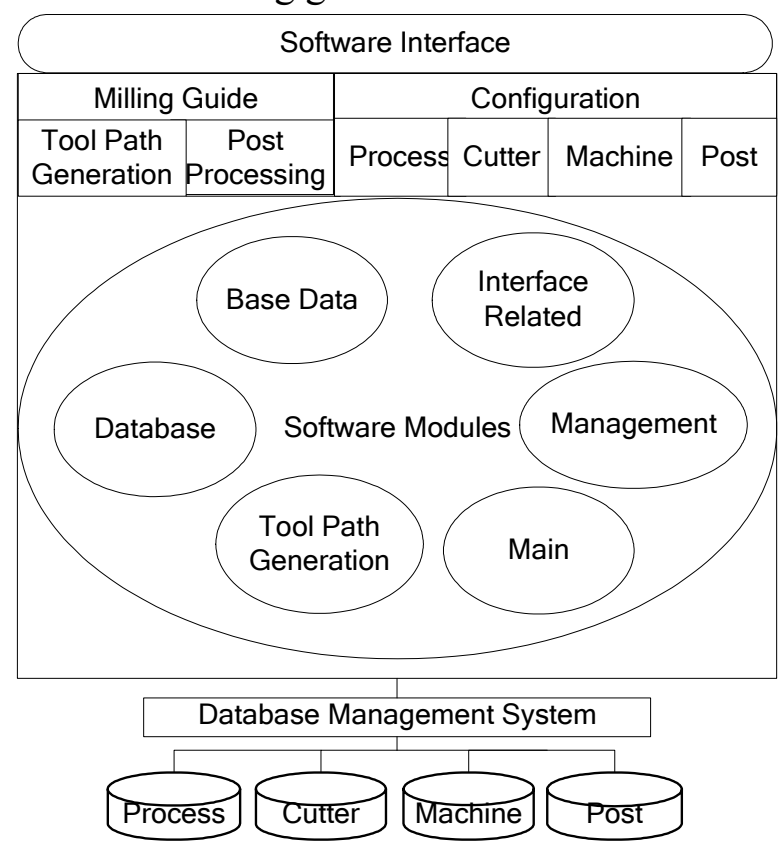

Fig.3 Software Module Illustration 


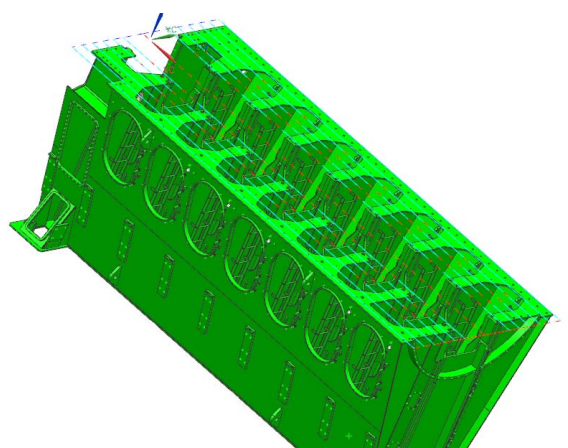

Fig.4 Tool Path Generated

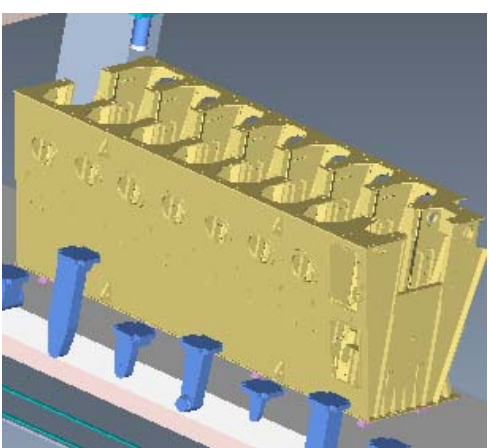

Fig.5 Vericut Simulation

System Running. During the system running, user can select the features to produce the correspondent path. Then the system will find the correct cutting arguments according to the information in template and database, so that appropriate tool path can be generated, which is illustrated in Fig.5. In the post treatment, suitable NC code would be produced by different customized postprocessor. As seen in Fig.4, the tool path simulation is done in Vericut to validate the correctness of the $\mathrm{NC}$ code.

\section{Summary}

The manufacture of key parts for marine diesel engine is focused in this paper. The similarity between features is used, and then the different feature information is categorized modularly and stored in the database. With the information, auto programming and verification on tool path have been made to affirm the feasibility and practicability of the method. With this method, it can be convenient for preparation work for production in plant; and the repeated workload for the manufacturing can be reduced as much as possible. And this modularization principle can be useful for similar complicated manufacturing production. In the future work, 3D annotation techniques can be used to embed the feature information into the 3D digital product model, and the modularization can be applied on CAD/CAPP/CAM integration system all on 3D digital product definition.

\section{References}

[1] DU Juan, Tian Xi-tian, ZHU Ming-quan, LIU Shu-nuan and LI Jian-ke. Integration Technology of $C A D / C A P P / C A M / C N C$ Systems Based on STEP \& STEP-NC. Computer Integrated manufacturing Systems, Vol.11 (2005), p. 42-45.

[2] ZHANG Sheng-wen, DING Yu-ling, WANG Gui-cheng, YANG Chang-qi and JIA Wei. CAD/CAPP/CAM Integration System for Frame Parts of Marine Diesel. Computer Integrated Manufacturing Systems, Vol.17 (2011), p. 760-766.

[3] Zhang Sheng-wen, Liu Yang and Fang Xi-feng. Research of Feature Recognition Method for Box Part Based on STEP. Proceedings of Measuring Technology and Mechatronics Automation. Zuerich, Switzerland: Trans Tech Publications Ltd, 2009, 108-113.

[4] Tong Shi-zhong. Current Situation and Progresss of the Study and Practice of Modularization. Electro-Mechanical Engineering. Vol.2 (2011), p. 1-8.

[5] Wu Zheng-gang, Han Yu-qi, Song Hua-ming and Meng Qing-liang. Research on the Modular Manufacturing Strategy for Complex Products. China Mechanical Engineering, Vol.16 (2005), p. 1826-1830.

[6] Young-Keun Choi and A. banerjee. Tool Path Generation and Tolerance Analysis for Free-form Surfaces. International Journal of Machine Tools \& Manufacture, Vol.47 (2007), p. 689-696.

[7] ZHANG Sheng-wen, ZHANG Rui-guo, JIA Wei and YANG Chang-qi. Automatic programming system for complex parts based on Group Technology. Manufacturing Technology and Machine Tool. Vol.03 (2010), p. 97-100.

[8] GRABOWIK C, KAL INOWSKI K and MONICA Z. Integration of CAD/CAPP/ PC system s. Journal of Materials Processing Technology, Vol.10 (2005), p. 1358- 1368. 